\title{
Right Invertible Multiplication Operators and Stable Rational Matrix Solutions to an Associate Bezout Equation, I: The Least Squares Solution
}

\author{
A. E. Frazho, M. A. Kaashoek, A. C. M. Ran
}

\begin{abstract}
In this paper a state space formula is derived for the least squares solution $X$ of the corona type Bezout equation $G(z) X(z)=I_{m}$. Here $G$ is a (possibly non-square) stable rational matrix function. The formula for $X$ is given in terms of the matrices appearing in a state space representation of $G$ and involves the stabilizing solution of an associate discrete algebraic Riccati equation. Using these matrices, a necessary and sufficient condition is given for right invertibility of the operator of multiplication by $G$. The formula for $X$ is easy to use in Matlab computations and shows that $X$ is a rational matrix function of which the McMillan degree is less than or equal to the McMillan degree of $G$.
\end{abstract}

Mathematics Subject Classification (2010). Primary 47B35, 39B42; Secondary 47A68, 93B28.

Keywords. Right invertible multiplication operator, Toeplitz operators, Bezout equation, Stable rational matrix functions, State space representation, Discrete algebraic Riccati equation, Stabilizing solution.

\section{Introduction}

Throughout this paper $G$ is a stable rational $m \times p$ matrix function. Here stable means that $G$ has all its poles in $|z|>1$, infinity included. In particular, $G$ is a rational matrix-valued $H^{\infty}$ function. In general, $p$ will be larger than $m$, and thus $G$ will be a "fat" non-square matrix function. We shall be dealing with the corona type Bezout equation

$$
G(z) X(z)=I_{m}, \quad z \in \mathbb{D} \text {. }
$$

Equation (1.1) — for arbitrary $H^{\infty}$ functions - has a long and interesting history, starting with Carleson's corona theorem [4] (for the case when $m=1$ )

The research of the first author was partially supported by a visitors grant from NWO (Netherlands Organisation for Scientific Research). 
and Fuhrmann's extension to the matrix-valued case [10]. The topic has beautiful connections with operator theory (see [1,22,24], the books [15,19-21], and the more recent papers [26-28]). Rational matrix equations of the form (1.1) also play an important role in solving systems and control theory problems, in particularly, in problems involving coprime factorization, see, e.g., [30, Section 4.1], [13, Section A.2], [31, Chapter 21]). For matrix polynomials (1.1) is closely related to the Sylvester resultant; see, e.g., Section 3 in [12] and the references in this paper.

The operator version of the corona theorem tells us that (1.1) has a $p \times m$ matrix valued $H^{\infty}$ solution $X$ if and only if the operator $M_{G}$ of multiplication by $G$ mapping the Hardy space $H^{2}\left(\mathbb{C}^{p}\right)$ into the Hardy space $H^{2}\left(\mathbb{C}^{m}\right)$ is right invertible. The necessity of this condition is trivial; sufficiency can be proved by using the commutant lifting theorem (see, e.g., [21, Theorem 3.6.1]). In our case, because $G(z)$ is rational, a simple approximation argument (see the paragraph after Proposition 2.1 below) shows that the existence of a $H^{\infty}$ solution implies the existence of a rational $H^{\infty}$ - solution.

Assuming that $M_{G}$ is right invertible, let $X$ be the $p \times m$ matrix function defined by

$$
X(\cdot) y=M_{G}^{*}\left(M_{G} M_{G}^{*}\right)^{-1} E y, \quad y \in \mathbb{C}^{m} .
$$

Here $E$ is the canonical embedding of $\mathbb{C}^{m}$ into $H^{2}\left(\mathbb{C}^{m}\right)$, that is, $(E y)(z)=y$ for each $z \in \mathbb{D}$ and $y \in \mathbb{C}^{m}$. We shall see (Theorem 1.1 or Proposition 2.1 below) that the function $X$ determined by (1.2) is a stable rational matrix function satisfying (1.1). Note that the operator $M_{G}^{*}\left(M_{G} M_{G}^{*}\right)^{-1}$ is the unique (Moore-Penrose) right inverse of $M_{G}$ mapping $H^{2}\left(\mathbb{C}^{m}\right)$ onto the orthogonal complement of $\operatorname{Ker} M_{G}$ in $H^{2}\left(\mathbb{C}^{p}\right)$. This implies that the solution $X$ of (1.1) defined by (1.2) has an additional minimality property, namely given a stable rational matrix solution $V$ of (1.1) we have

$$
\|X(\cdot) u\|_{H^{2}\left(\mathbb{C}^{p}\right)} \leq\|V(\cdot) u\|_{H^{2}\left(\mathbb{C}^{p}\right)} \text { for each } u \text { in } \mathbb{C}^{m},
$$

or equivalently

$$
\frac{1}{2 \pi} \int_{0}^{2 \pi} X\left(e^{i t}\right)^{*} X\left(e^{i t}\right) d t \leq \frac{1}{2 \pi} \int_{0}^{2 \pi} V\left(e^{i t}\right)^{*} V\left(e^{i t}\right) d t .
$$

Moreover, equality holds in (1.4) if and only if $V=X$. For this reason we refer to the matrix function $X$ defined by (1.2) as the least squares solution of (1.1). We note that the use of the Moore-Penrose right inverse $M_{G}^{*}\left(M_{G} M_{G}^{*}\right)^{-1}$ is not uncommon in the analysis of the corona problem (see, e.g., Section 1 in [27]).

Let us now describe the main result of the present paper. The starting point is a state space representation of $G$. As is well-known from mathematical systems theory, the fact that $G$ is a stable rational matrix function, allows us (see, e.g., Chapter 1 of [5] or Chapter 4 in [2]) to write $G$ in the following form:

$$
G(z)=D+z C\left(I_{n}-z A\right)^{-1} B
$$


Here $A, B, C, D$ are matrices of appropriate sizes, $I_{n}$ is an identity matrix of order $n$, and the $n \times n$ matrix $A$ is stable, that is, $A$ has all its eigenvalues in the open unit disc $\mathbb{D}$. In the sequel we shall refer to the right hand side of (1.5) as a stable state space representation. State space representations are not unique. By definition the smallest $n$ for which $G$ has a stable state space representation of the form (1.5) is called the McMillan degree of $G$, denoted by $\delta(G)$. From the stability of the matrix $A$ in (1.5) it follows that the symmetric Stein equation

$$
P-A P A^{*}=B B^{*}
$$

has a unique solution $P$. Given this $n \times n$ matrix $P$ we introduce two auxiliary matrices:

$$
R_{0}=D D^{*}+C P C^{*}, \quad \Gamma=B D^{*}+A P C^{*} .
$$

The following theorem is our main result.

Theorem 1.1. Let $G$ be the $m \times p$ rational matrix function given by the stable state space representation (1.5). Let $P$ be the unique solution of the Stein equation (1.6), and let the matrices $R_{0}$ and $\Gamma$ be given by (1.7). Then equation (1.1) has a stable rational matrix solution if and only if

(i) the discrete algebraic Riccati equation

$$
Q=A^{*} Q A+\left(C-\Gamma^{*} Q A\right)^{*}\left(R_{0}-\Gamma^{*} Q \Gamma\right)^{-1}\left(C-\Gamma^{*} Q A\right)
$$

has a (unique) stabilizing solution $Q$, that is, $Q$ is an $n \times n$ matrix with the following properties:

(a) $R_{0}-\Gamma^{*} Q \Gamma$ is positive definite,

(b) $Q$ satisfies the Riccati equation (1.8),

(c) the matrix $A-\Gamma\left(R_{0}-\Gamma^{*} Q \Gamma\right)^{-1}\left(C-\Gamma^{*} Q A\right)$ is stable;

(ii) the matrix $I_{n}-P Q$ is non-singular.

Moreover, (i) and (ii) are equivalent to $M_{G}$ being right invertible. Furthermore, if (i) and (ii) hold, then the $p \times m$ matrix-valued function $X$ defined by (1.2) is a stable rational matrix solution of (1.1) and $X$ admits the following the state space representation:

$$
X(z)=\left(I_{p}-z C_{1}\left(I_{n}-z A_{0}\right)^{-1}\left(I_{n}-P Q\right)^{-1} B\right) D_{1},
$$

where

$$
\begin{aligned}
& A_{0}=A-\Gamma\left(R_{0}-\Gamma^{*} Q \Gamma\right)^{-1}\left(C-\Gamma^{*} Q A\right), \\
& C_{1}=D^{*} C_{0}+B^{*} Q A_{0}, \text { with } C_{0}=\left(R_{0}-\Gamma^{*} Q \Gamma\right)^{-1}\left(C-\Gamma^{*} Q A\right), \\
& D_{1}=\left(D^{*}-B^{*} Q \Gamma\right)\left(R_{0}-\Gamma^{*} Q \Gamma\right)^{-1}+C_{1}\left(I_{n}-P Q\right)^{-1} P C_{0}^{*} .
\end{aligned}
$$

Finally, $X$ is the least squares solution of (1.1), the McMillan degree of $X$ is less than or equal to the McMillan degree of $G$, and

$$
\frac{1}{2 \pi} \int_{0}^{2 \pi} X\left(e^{i t}\right)^{*} X\left(e^{i t}\right) d t=D_{1}^{*}\left(I_{p}+B^{*} Q\left(I_{n}-P Q\right)^{-1} B\right) D_{1} .
$$


The necessary and sufficient state space conditions for the existence of a stable rational matrix solution and the formula for the least squares solution given in the above theorem are new. They resemble analogous conditions and formulas appearing in the state space theory of discrete $H^{2}$ and $H^{\infty}$ optimal control; see [16,17,23], Chapter 21 in the book [31], see also [6] for the continuous time analogues. However, the algebraic Riccati equation in Theorem 1.1 is of the stochastic realization type with the solution $Q$ being positive semidefinite, while the $H^{\infty}$ or $H^{2}$ control Riccati equations in the mentioned references are of the LQR type again with the solutions being positive semidefinite (see, e.g., [14, Chapter 5] for the LQR type, and [14, Chapter 6] for the stochastic realization type). It is easy to rewrite the stochastic realization Riccati equation into the LQR type, but then the condition on the stabilizing solution being positive semidefinite changes into negative semidefinite. As far as we know there is no direct way to reduce the problem considered in the present paper to a standard $H^{2}$ control problem or to a coprime factorization problem. Concerning the latter, the discrete time analogue of the coprime method employed in [30, Section 4.1] could be used to obtain a parametrization of all stable rational solutions of (1.1). However, minimal $H^{2}$-solutions are not considered in [30], and to the best of our knowledge coprime factorization does not provide a method to single out such a solution. Moreover, it is not clear whether or not the minimal $H^{2}$-solution $X$ considered in the present paper does appear among the solutions obtained by using the discrete time analogue of the state space formulas given in [30, Section 4.2]; see the final part of Example 2 in Sect. 5 for a negative result in this direction.

We remark that Theorem 1.1 provides a computationally feasible way to check whether or not for a given $m \times p$ stable rational matrix function $G$ the multiplication operator $M_{G}$ is right invertible and to obtain the least squares solution in that case. Indeed, first one constructs a realization (1.5) in the standard way. Next, one solves (1.6) for $P$, for instance by using the Matlab command dgram or dlyap. With $P$ one constructs the matrices $R_{0}$ and $\Gamma$ as in (1.7). Then solve the algebraic Riccati equation (1.8) for $Q$, either using the Matlab command dare or an iterative method. Finally, one checks that one is not an eigenvalue of $P Q$. Continuing in this way one also computes the least squares solution $X$ given by (1.9).

In the subsequent paper [9], assuming $M_{G}$ is right invertible, we shall present a state space description of the set of all stable rational matrix solutions of equation (1.1) and a full description of the null space of $M_{G}$. In that second paper we shall also discuss the connection with the related Tolokonnikov lemma [25] for the rational case.

The paper consists of five sections, the first being the present introduction. Sections 2 and 3 have a preparatory character. The basic operator theory results on which Theorem 1.1 is based are presented in Sect. 2. In Sect. 3 we explain the role of the stabilizing solution $Q$ of the Riccati equation appearing in Theorem 1.1. Also a number of auxiliary state space formulas are presented in this third section. The proof of Theorem 1.1 is given in Sect. 4. In Sect. 5 we present two examples, and illustrate the comment on MatLab procedures made above. 


\section{The Underlying Operator Theory Results}

We begin with some terminology and notation. Let $F$ be any $m \times p$ matrix-valued function of which the entries are essentially bounded on the unit circle $\mathbb{T}$. Recall (see, e.g., Chapter XXIII in [11]) that the block Toeplitz operator defined by $F$ is the operator $T_{F}$ given by

$$
T_{F}=\left[\begin{array}{cccc}
F_{0} & F_{-1} & F_{-2} & \cdots \\
F_{1} & F_{0} & F_{-1} & \cdots \\
F_{2} & F_{1} & F_{0} & \cdots \\
\vdots & \vdots & \vdots & \ddots
\end{array}\right]: \ell_{+}^{2}\left(\mathbb{C}^{p}\right) \rightarrow \ell_{+}^{2}\left(\mathbb{C}^{m}\right) .
$$

Here ..., $F_{-1}, F_{0}, F_{1}, \ldots$ are the block Fourier coefficients of $F$. By $H_{F}$ we denote the block Hankel operator determined by the block Fourier coefficients $F_{j}$ with $j=1,2, \ldots$, that is,

$$
H_{F}=\left[\begin{array}{cccc}
F_{1} & F_{2} & F_{3} & \cdots \\
F_{2} & F_{3} & F_{4} & \cdots \\
F_{3} & F_{4} & F_{5} & \cdots \\
\vdots & \vdots & \vdots & \ddots
\end{array}\right]: \ell_{+}^{2}\left(\mathbb{C}^{p}\right) \rightarrow \ell_{+}^{2}\left(\mathbb{C}^{m}\right) .
$$

We shall write $\tilde{E}$ for the canonical embedding of $\mathbb{C}^{m}$ onto the first coordinate space of $\ell_{+}^{2}\left(\mathbb{C}^{m}\right)$. Note that $T_{F}^{*} \tilde{E}$ is just equal to the operator from $\mathbb{C}^{m}$ into $\ell_{+}^{2}\left(\mathbb{C}^{p}\right)$ defined by the first column of $T_{F}^{*}$. The identity operator on $\ell_{+}^{2}\left(\mathbb{C}^{m}\right)$ or $\ell_{+}^{2}\left(\mathbb{C}^{p}\right)$ will be denoted by $I$. The symbol $I_{n}$ stands $n \times n$ identity matrix or the identity operator on $\mathbb{C}^{n}$.

Let $G$ be a stable rational $m \times p$ matrix function. In this case $H_{G}$ is an operator of finite rank and its rank is equal to the McMillan degree $\delta(G)$. Furthermore, the multiplication operator $M_{G}$ used in the previous section is unitarily equivalent to the block Toeplitz operator $T_{G}$. In fact, $M_{G} \mathcal{F}_{\mathbb{C}^{p}}=$ $\mathcal{F}_{\mathbb{C}^{m}} T_{G}$, where for each positive integer $k$ the operator $\mathcal{F}_{\mathbb{C}^{k}}$ is the Fourier transform mapping $\ell_{+}^{2}\left(\mathbb{C}^{k}\right)$ onto the Hardy space $H^{2}\left(\mathbb{C}^{k}\right)$. In what follows it will be more convenient to work with $T_{G}$ than with $M_{G}$. Note that $\mathcal{F}_{\mathbb{C}^{m}} \tilde{E}=E$, where $E$ is the embedding operator appearing in (1.2). Furthermore, the expression $M_{G}\left(M_{G} M_{G}^{*}\right)^{-1}$, also appearing in (1.2), can be derived from

$$
M_{G}^{*}\left(M_{G} M_{G}^{*}\right)^{-1} \mathcal{F}_{\mathbb{C}^{m}}=\mathcal{F}_{\mathbb{C}^{p}} T_{G}^{*}\left(T_{G} T_{G}^{*}\right)^{-1} .
$$

The following result provides the operator theory background for the proof of Theorem 1.1.

Proposition 2.1. Let $G$ be a stable rational $m \times p$ matrix function, and let $R$ be the rational $m \times m$ matrix function given by $R(z)=G(z) G^{*}(z)$. Then the following four statements are equivalent.

(a) The equation $G X=I$ has a stable rational matrix solution.

(b) The Toeplitz operator $T_{G}$ is right invertible.

(c) The Toeplitz operator $T_{R}$ is invertible and the same holds true for the operator $I-H_{G}^{*} T_{R}^{-1} H_{G}$. 
Moreover, if one of these conditions is satisfied, then $T_{G} T_{G}^{*}$ is invertible, its inverse is given by

$$
\left(T_{G} T_{G}^{*}\right)^{-1}=T_{R}^{-1}+T_{R}^{-1} H_{G}\left(I-H_{G}^{*} T_{R}^{-1} H_{G}\right)^{-1} H_{G}^{*} T_{R}^{-1},
$$

and the function $X=\mathcal{F}_{\mathbb{C}^{p}} T_{G}^{*}\left(T_{G} T_{G}^{*}\right)^{-1} \tilde{E}$ is a stable rational matrix function satisfying (1.1).

We note that the equivalence of (a) and (b) in the above proposition is known. In fact, (a) implies (b) is trivial, and (b) implies the existence of a $H^{\infty}$ solution. But if (1.1) has an $H^{\infty}$ solution, then it also has a stable rational matrix solution. The latter follows from a simple approximation argument. To see this, given an $H^{\infty}$ function $F$ and $0<r<1$, let us write $F_{r}$ for the function $F_{r}(z)=F(r z)$. Now assume that $X$ is an $H^{\infty}$ solution of (1.1). Then $G_{r}(z) X_{r}(z)=I_{m}$, and hence

$$
G(z) X_{r}(z)=I_{m}-\left(G(z)-G_{r}(z)\right) X_{r}(z), \quad|z|<1 .
$$

Since $G$ is rational, $G_{r}(z) \rightarrow G(z)$ uniformly on $|z| \leq 1$ for $r \rightarrow \infty$. Furthermore, $\left\|X_{r}\right\|_{\infty} \rightarrow\|X\|_{\infty}$ for $r \rightarrow \infty$, and the sequence $\left\{\left\|X_{r}\right\|_{\infty}\right\}_{r \geq 1}$ is uniformy bounded. Thus there exists $r_{\circ}$ such that $\left\|\left(G-G_{r_{\circ}}\right) X_{r_{\circ}}\right\|_{\infty}<1 / 2$. Since $X_{r_{\circ}}(z)$ is continuous on $|z| \leq 1$, there exists a stable rational matrix function $\tilde{X}$ such that $\left\|X_{r_{\circ}}-\tilde{X}\right\|_{\infty}$ is strictly less than $\left(4+4\|G\|_{\infty}\right)^{-1}$. Now note that

$G(z) \tilde{X}(z)=I_{m}+\left(G(z)-G_{r_{\circ}}(z)\right) X_{r_{\circ}}(z)-G(z)\left(X_{r_{\circ}}(z)-\tilde{X}(z)\right), \quad|z|<1$.

Moreover, $\left\|\left(G-G_{r_{\circ}}\right) X_{r_{\circ}}\right\|_{\infty}+\left\|G\left(X_{r_{\circ}}-\tilde{X}\right)\right\|_{\infty}<3 / 4$. Hence $G \tilde{X}$ is a stable rational matrix function which has a stable rational matrix inverse. This implies that $\tilde{X}(G \tilde{X})^{-1}$ is a stable rational matrix solution of (1.1). ${ }^{1}$

In order to prove Proposition 2.1 it will be convenient to prove the following lemma first.

Lemma 2.2. Let $G$ be a stable rational $m \times p$ matrix function, and let $R$ be the rational $m \times m$ matrix function given by $R(z)=G(z) G^{*}(z)$. Assume $T_{R}$ is invertible. Then $T_{G}$ has closed range, the spectrum of $H_{G}^{*} T_{R}^{-1} H_{G}$ is contained in the closed interval $[0,1]$, and

$$
\operatorname{dim} \operatorname{Ker} T_{G}^{*}=\operatorname{dim} \operatorname{Ker}\left(I-H_{G}^{*} T_{R}^{-1} H_{G}\right)<\infty .
$$

In particular, $T_{G}$ is semi-Fredholm.

Proof. We shall need the identity

$$
T_{R}=T_{G G^{*}}=T_{G} T_{G}^{*}+H_{G} H_{G}^{*} .
$$

This identity can be found, for example, in [11], see formula (4) in Section XXIII.4 of [11]. It was proved there for the case when the entries of $T_{G}$ and $H_{G}$ are square matrices, but the general case can be reduced to the square case by adding zero rows or columns to the entries. Since $T_{R}$ is assumed to be invertible, (2.6) yields

$$
T_{G} T_{G}^{*}=T_{R}-H_{G} H_{G}^{*}=T_{R}\left(I-T_{R}^{-1} H_{G} H_{G}^{*}\right) .
$$

\footnotetext{
${ }^{1}$ We thank the referee for providing the above argument.
} 
Recall that $H_{G}$ has finite rank. Thus the first equality in (2.7) shows that $T_{G} T_{G}^{*}$ is a finite rank perturbation of an invertible operator. Hence $T_{G} T_{G}^{*}$ is a Fredholm operator of index zero. As is well-known, the latter implies that $T_{G}$ has closed range (cf., Exercise 2 on page 283 of [11]).

Next we use the fact that $\operatorname{Ker} T_{G}$ is perpendicular to $\operatorname{Im} T_{G}^{*}$. This implies that the operator $T_{G}$ is one-to-one on $\operatorname{Im} T_{G}^{*}$, and therefore $\operatorname{Ker} T_{G}^{*}=$ $\operatorname{Ker} T_{G} T_{G}^{*}$. Since is $\operatorname{dim} \operatorname{Ker} T_{G} T_{G}^{*}$ is finite, the same holds true for $\operatorname{dim} \operatorname{Ker} T_{G}^{*}$. Furthermore, we can use the second identity in (2.7) to show that

$$
\begin{aligned}
\operatorname{dim} \operatorname{Ker} T_{G}^{*} & =\operatorname{dim} \operatorname{Ker} T_{G} T_{G}^{*}=\operatorname{dim} \operatorname{Ker} T_{R}\left(I-T_{R}^{-1} H_{G} H_{G}^{*}\right) \\
& =\operatorname{dim} \operatorname{Ker}\left(I-T_{R}^{-1} H_{G} H_{G}^{*}\right) \\
& =\operatorname{dim} \operatorname{Ker}\left(I-H_{G}^{*} T_{R}^{-1} H_{G}\right) .
\end{aligned}
$$

This proves (2.5).

It remains to prove that the spectrum of $H_{G}^{*} T_{R}^{-1} H_{G}$ is contained in the closed interval $[0,1]$. Since $H_{G}^{*} T_{R}^{-1} H_{G}$ is selfadjoint, it suffices to show that the spectral radius of $H_{G}^{*} T_{R}^{-1} H_{G}$ is at most one. To do this we use the fact that $T_{R}$ is strictly positive, which implies that $T_{R}$ factors as $T_{R}=\Lambda^{*} \Lambda$, with $\Lambda$ being an invertible operator. For instance, for $\Lambda$ we can take the square root of $T_{R}$. Multiplying (2.6) from the left by $\Lambda^{-1}$ and from the right by $\Lambda^{-*}$ yields the identity

$$
I-\Lambda^{-*} H_{G} H_{G}^{*} \Lambda^{-1}=\Lambda^{-*} T_{G} T_{G}^{*} \Lambda^{-1} .
$$

The right hand side of the latter identity is non-negative, and hence the operator $\Lambda^{-*} H_{G} H_{G}^{*} \Lambda^{-1}$ is a contraction. In particular, its spectrum is in the closed unit disc, that is, $r_{\text {spec }}\left(\Lambda^{-*} H_{G} H_{G}^{*} \Lambda^{-1}\right) \leq 1$. Here $r_{\text {spec }}(K)$ stands for the spectral radius of the operator $K$. But the spectral radius of a product of two operators is independent of the order of the operators. Thus

$$
\begin{aligned}
r_{\text {spec }}\left(H_{G}^{*} T_{R}^{-1} H_{G}\right) & =r_{\text {spec }}\left(\left(H_{G}^{*} \Lambda^{-1}\right)\left(\Lambda^{-*} H_{G}\right)\right) \\
& =r_{\text {spec }}\left(\Lambda^{-*} H_{G} H_{G}^{*} \Lambda^{-1}\right) .
\end{aligned}
$$

We conclude $r_{\text {spec }}\left(H_{G}^{*} T_{R}^{-1} H_{G}\right) \leq 1$, as desired.

Proof of Proposition 2.1. We split the proof into three parts. The equivalence (a) $\Rightarrow$ (b) is trivial. The first part of the proof deals with (b) $\Rightarrow$ (c). In the second part, assuming (c) holds, we derive (2.4), and in the third part, again assuming (c), we prove the final statement of the theorem and (c) $\Rightarrow$ (a). On the way we give a new proof of (b) $\Rightarrow$ (a) not using the corona theorem as was done in the paragraph directly after Proposition 2.1.

Part 1. Assume that $T_{G}$ is right invertible. Then $T_{G} T_{G}^{*}$ is strictly positive. As $H_{G} H_{G}^{*}$ is non-negative, it follows from (2.6) that $T_{R}$ is strictly positive. In particular, $T_{R}$ is invertible. Since $H_{G}^{*} T_{R}^{-1} H_{G}$ is a finite rank operator, $I-H_{G}^{*} T_{R}^{-1} H_{G}$ is invertible if and only if $I-H_{G}^{*} T_{R}^{-1} H_{G}$ is one-to-one. The fact that $T_{G}$ is right invertible implies that $\operatorname{Ker} T_{G}^{*}$ consists of the zero element only, and hence formula (2.5) shows that $I-H_{G}^{*} T_{R}^{-1} H_{G}$ is indeed one-to-one. Thus $I-H_{G}^{*} T_{R}^{-1} H_{G}$ invertible, and (c) is proved. 
Part 2. In this part we assume (c) and derive (2.4). Assume that $T_{R}$ is invertible and that the same holds true for the operator $I-H_{G}^{*} T_{R}^{-1} H_{G}$. Hence we can apply Lemma 2.2 to show that $T_{G}^{*}$ is a one-to-one operator with closed range. This implies that $T_{G}$ is surjective, and hence $T_{G} T_{G}^{*}$ is invertible. But then we can use (2.7) to show that

$$
\begin{aligned}
\left(T_{G} T_{G}^{*}\right)^{-1} & =\left(I-T_{R}^{-1} H_{G} H_{G}^{*}\right)^{-1} T_{R}^{-1} \\
& =\left(I+T_{R}^{-1} H_{G}\left(I-H_{G}^{*} T_{R}^{-1} H_{G}\right)^{-1} H_{G}^{*}\right) T_{R}^{-1} \\
& =T_{R}^{-1}+T_{R}^{-1} H_{G}\left(I-H_{G}^{*} T_{R}^{-1} H_{G}\right)^{-1} H_{G}^{*} T_{R}^{-1} .
\end{aligned}
$$

Thus the inverse of $T_{G} T_{G}^{*}$ is given by (2.4). Note that the above also shows $(\mathrm{c}) \Rightarrow(\mathrm{b})$, and thus (b) and (c) are equivalent.

Part 3. In this part we assume (c) holds and derive (a). To do this it remains to prove the final statement of the theorem. For this purpose we need the following terminology. A vector $x$ in $\ell_{+}^{2}\left(\mathbb{C}^{m}\right)$ is said to be a rational vector whenever $\mathcal{F}_{\mathbb{C}^{m}} x$ is a stable rational $m \times 1$ matrix function. If $F$ is a rational $m \times p$ matrix function without poles on the unit circle $\mathbb{T}$, then $T_{F}$ maps rational vectors in $\ell_{+}^{2}\left(\mathbb{C}^{p}\right)$ into rational vectors in $\ell_{+}^{2}\left(\mathbb{C}^{m}\right)$ and the range of $H_{F}$ consists of rational vectors only. These facts are well-known; for the statement about the range of $H_{F}$ see the remark made at the end of the second paragraph of Sect. 3 .

We first show that $\left(T_{G} T_{G}^{*}\right)^{-1}$ maps rational vectors into rational vectors. To do this, let $x$ be a rational vector in $\ell_{+}^{2}\left(\mathbb{C}^{m}\right)$. Put

$$
y=H_{G}\left(I-H_{G}^{*} T_{R}^{-1} H_{G}\right)^{-1} H_{G}^{*} T_{R}^{-1} x .
$$

Thus $\left(T_{G} T_{G}^{*}\right)^{-1} x=T_{R}^{-1}(x+y)$. Since $G$ is a stable rational matrix function and $y$ is in the range of $H_{G}$, we know (see the previous paragraph) that $y$ is a rational vector. Thus we have to show $T_{R}^{-1}(x+y)$ is a rational vector. Note that $x+y$ is a rational vector. As $R$ is positive definite on the unit circle, $R$ admits a spectral factorization relative to the unit circle. It follows that $T_{R}^{-1}$ can be written as $T_{R}^{-1}=T T^{*}$ where $T$ is a Toeplitz operator defined by a stable rational matrix function (see Theorem 3.2 below for more details). Thus both $T$ and $T^{*}$ are Toeplitz operators defined by a rational matrix function without poles on the unit circle. But such Toeplitz operators map rational vectors into rational vectors (see the previous paragraph). We conclude that $T_{R}^{-1}(x+y)$ is a rational vector, and thus $\left(T_{G} T_{G}^{*}\right)^{-1} x$ is a rational vector.

Now put

$$
\tilde{\Xi}=\left(T_{G} T_{G}^{*}\right)^{-1} \tilde{E} \quad \text { and } \quad \tilde{X}=T_{G}^{*}\left(T_{G} T_{G}^{*}\right)^{-1} \tilde{E} .
$$

From the result of the previous paragraph we know that for each $u$ in $\mathbb{C}^{p}$ the vector $\tilde{\Xi} u$ is a rational vector in $\ell_{+}^{2}\left(\mathbb{C}^{p}\right)$. Note that $\tilde{X} u=T_{G}^{*} \tilde{\Xi} u$, and recall that a Toeplitz operator defined by a rational matrix function maps rational vectors into rational vectors. Hence $\tilde{X} u$ is also a rational vector. This implies that $X=\mathcal{F}_{\mathbb{C}^{p}} \tilde{X}$ is a stable rational matrix function. From

$$
T_{G} \tilde{X}=T_{G} T_{G}^{*}\left(T_{G} T_{G}^{*}\right)^{-1} \tilde{E}=\tilde{E}
$$

it follows that $G(z) X(z)=I_{m}$. Thus (a) holds and the final statements of the theorem are proved. 
Both Proposition 2.1 and Lemma 2.2 hold in greater generality. For instance, Lemma 2.2 remains true when $G$ is an $m \times p$ matrix-valued $H^{\infty}$ function continuous on the closed unit disk. Also the equivalence of (a), (b) and (c) in Proposition 2.1 as well as formula (2.4) remain true for such a function $G$, provided one allows in (a) for $H^{\infty}$ solutions.

\section{Preliminaries About the Riccati Equation}

In this section we clarify the role of the Riccati equation (1.8), and present some auxiliary state space formulas. Throughout this and the following sections we assume that $G$ is given by the stable state space representation (1.5). With this representation we associate the operators

$$
\begin{aligned}
W_{\text {obs }} & =\left[\begin{array}{c}
C \\
C A \\
C A^{2} \\
\vdots
\end{array}\right]: \mathbb{C}^{n} \rightarrow \ell_{+}^{2}\left(\mathbb{C}^{m}\right) \\
W_{\text {con }} & =\left[\begin{array}{llll}
B & A B & A^{2} B & A^{3} B \cdots
\end{array}\right]: \ell_{+}^{2}\left(\mathbb{C}^{p}\right) \rightarrow \mathbb{C}^{n} .
\end{aligned}
$$

The fact that the matrix $A$ is stable implies that these operators are welldefined and bounded. We call $W_{\text {obs }}$ the observability operator and $W_{\text {con }}$ the controllability operator corresponding to the state space representation (1.5).

Since for $j=1,2, \ldots$ the $j$-th Taylor coefficient of $G$ at zero is given by $C A^{j-1} B$ it follows from (3.1) and (3.2) that

$$
H_{G}=\left[\begin{array}{cccc}
G_{1} & G_{2} & G_{3} & \cdots \\
G_{2} & G_{3} & G_{4} & \cdots \\
G_{3} & G_{4} & G_{5} & \cdots \\
\vdots & \vdots & \vdots & \ddots
\end{array}\right]=W_{o b s} W_{c o n} .
$$

From (3.3) it is clear that $\operatorname{rank} H_{G}$ is finite and the range of $H_{G}$ consists of rational vectors.

Recall that $P$ is the unique solution of the Stein equation

$$
P-A P A^{*}=B B^{*} .
$$

Thus $P=\sum_{\nu=0}^{\infty} A^{\nu} B B^{*}\left(A^{*}\right)^{\nu}=W_{c o n} W_{c o n}^{*}$, where $W_{\text {con }}$ is defined by (3.2). Recall that $P$ is unique because $A$ is stable.

Lemma 3.1. Let $G$ be the $m \times p$ rational matrix function given by the stable state space representation (1.5), and let $P$ be the unique solution of the Stein equation (3.4). Put $R(z)=G(z) G^{*}(z)$, where $G^{*}(z)=G\left(\bar{z}^{-1}\right)^{*}$. Then $R$ admits the following representation

$$
R(z)=z C\left(I_{n}-z A\right)^{-1} \Gamma+R_{0}+\Gamma^{*}\left(z I_{n}-A^{*}\right)^{-1} C^{*},
$$

where

$$
R_{0}=D D^{*}+C P C^{*}, \quad \Gamma=B D^{*}+A P C^{*} .
$$


Proof. From (1.5) we see that

$$
R(z)=G(z) G^{*}(z)=G(z) D^{*}+G(z) B^{*}\left(z I_{n}-A^{*}\right)^{-1} C^{*} .
$$

We first prove that

$$
G(z) B^{*}\left(z I_{n}-A^{*}\right)^{-1}=C\left(I_{n}-z A\right)^{-1} P+\Gamma^{*}\left(z I_{n}-A^{*}\right)^{-1} .
$$

To do this observe that

$$
\begin{aligned}
G(z) B^{*}\left(z I_{n}-A^{*}\right)^{-1}= & D B^{*}\left(z I_{n}-A^{*}\right)^{-1} \\
& +z C\left(I_{n}-z A\right)^{-1} B B^{*}\left(z I_{n}-A^{*}\right)^{-1} .
\end{aligned}
$$

From (3.4) we see that $z B B^{*}=P\left(z I_{n}-A^{*}\right)+\left(I_{n}-z A\right) P A^{*}$, and thus

$$
z\left(I_{n}-z A\right)^{-1} B B^{*}\left(z I_{n}-A^{*}\right)^{-1}=\left(I_{n}-z A\right)^{-1} P+P A^{*}\left(z I_{n}-A^{*}\right)^{-1} .
$$

Inserting the latter identity in the formula for $G(z) B^{*}\left(z I_{n}-A^{*}\right)^{-1}$ we obtain

$$
\begin{aligned}
G(z) B^{*}\left(z I_{n}-A^{*}\right)^{-1}= & D B^{*}\left(z I_{n}-A^{*}\right)^{-1} \\
& +C\left(I_{n}-z A\right)^{-1} P+C P A^{*}\left(z I_{n}-A^{*}\right)^{-1} .
\end{aligned}
$$

From the second identity in (3.6) we know that $\Gamma^{*}=D B^{*}+C P A^{*}$. Thus (3.8) holds.

Using the representation (1.5) and inserting (3.8) in (3.7) yields

$$
\begin{aligned}
G(z) G^{*}(z)= & G(z) D^{*}+C\left(I_{n}-z A\right)^{-1} P C^{*}+\Gamma^{*}\left(z I_{n}-A^{*}\right)^{-1} C^{*} \\
= & G(z) D^{*}+C P C^{*}+z C\left(I_{n}-z A\right)^{-1} A P C^{*}+\Gamma^{*}\left(z I_{n}-A^{*}\right)^{-1} C^{*} \\
= & D D^{*}+C P C^{*}+z C\left(I_{n}-z A\right)^{-1}\left(B D^{*}+A P C^{*}\right) \\
& +\Gamma^{*}\left(z I_{n}-A^{*}\right)^{-1} C^{*} .
\end{aligned}
$$

But $D D^{*}+C P C^{*}=R_{0}$ and $B D^{*}+A P C^{*}=\Gamma$ by (3.6). Thus (3.5) is proved.

Following [8] we associate with the representation (3.5) the discrete algebraic Riccati equation

$$
Q=A^{*} Q A+\left(C-\Gamma^{*} Q A\right)^{*}\left(R_{0}-\Gamma^{*} Q \Gamma\right)^{-1}\left(C-\Gamma^{*} Q A\right) .
$$

Note that this is precisely the Riccati equation appearing Theorem 1.1. Using the symmetric version of Theorem 1.1 in [8] (see Section 14.7 in [3] or Sections 10.2 and 10.2 in [7]) we know that $R(z)=G(z) G^{*}(z)$ is positive definite for each $z$ on the unit circle $\mathbb{T}$ if and only if the Riccati equation (3.9) has a solution $Q$ satisfying

(a) $R_{0}-\Gamma^{*} Q \Gamma$ is positive definite,

(b) $Q$ satisfies the Riccati equation (3.9),

(c) the matrix $A-\Gamma\left(R_{0}-\Gamma^{*} Q \Gamma\right)^{-1}\left(C-\Gamma^{*} Q A\right)$ is stable.

Moreover, this solution is unique and hermitian. In fact,

$$
Q=W_{o b s}^{*} T_{R}^{-1} W_{o b s} .
$$

Here $T_{R}$ is the block Toeplitz operator on $\ell_{+}^{2}\left(\mathbb{C}^{p}\right)$ defined by the matrix function $R$, and $W_{o b s}$ is defined by (3.2). The solution $Q$ satisfying (a), (b), (c) above will be called the stabilizing solution of (3.9), cf., Section 13.5 in [18]. 
In sequel, given the stabilizing solution $Q$ of (3.9), we write

$$
A_{0}=A-\Gamma C_{0}, \quad \text { where } C_{0}=\left(R_{0}-\Gamma^{*} Q \Gamma\right)^{-1}\left(C-\Gamma^{*} Q A\right) .
$$

Note that (c) tells us that $A_{0}$ is stable.

When (3.9) has a stabilizing $Q$, then (and only then) the function $R$ admits a right spectral factorization relative to the unit circle $\mathbb{T}$. Moreover, in that case, a right spectral factorization $R(z)=\Phi^{*}(z) \Phi(z)$ is obtained (see, e.g., Section 14.7 in [3]) by taking

$$
\Phi(z)=\Delta+z \Delta C_{0}\left(I_{n}-z A\right)^{-1} \Gamma, \quad \text { where } \quad \Delta=\left(R_{0}-\Gamma^{*} Q \Gamma\right)^{1 / 2} .
$$

Note that $\Delta$ is invertible, because $R_{0}-\Gamma^{*} Q \Gamma$ is invertible. The first identity in (3.11) then implies (cf., Theorem 2.1 in [2]) that

$$
\Phi(z)^{-1}=\Delta^{-1}-z C_{0}\left(I_{n}-z A_{0}\right)^{-1} \Gamma \Delta^{-1} .
$$

Since $A$ and $A_{0}$ are both stable, (3.12) and (3.13) both present stable state space representations, and hence $\Phi$ is invertible outer. (We call a square matrix-valued $H^{\infty}$ function $F$ invertible outer whenever $F(z)^{-1}$ exists and is again an $H^{\infty}$ function. Thus a square stable rational matrix function $F$ is invertible outer whenever $F(z)^{-1}$ exists and is a stable rational matrix function, i.e., $F$ is invertible in the algebra of stable square rational matrix functions.) Given the right spectral factorization $R(z)=\Phi^{*}(z) \Phi(z)$ with $\Phi$ given by (3.12), the block Toeplitz operator $T_{R}$ factors as $T_{R}=L^{*} L$, where $L=T_{\Phi}$. Note that both $T_{\Phi}$ and $T_{\Phi}^{-1}$ are block lower triangular. We summarize the above results in the following theorem.

Theorem 3.2. Let $G$ be given by (1.5) with $A$ stable, and put $R(z)=$ $G(z) G^{*}(z)$. Then $T_{R}$ is invertible if and only if the Riccati equation (3.9) has a stabilizing solution $Q$. In that case, $Q$ is uniquely determined by (3.10) and the inverse of $T_{R}$ is given by $T_{R}^{-1}=T_{\Psi} T_{\Psi}^{*}$. Here $T_{\Psi}$ is the block lower triangular Toeplitz operator on $\ell_{+}^{2}\left(\mathbb{C}^{m}\right)$ defined by the stable rational matrix function

$$
\Psi(z)=\left(I_{m}-z C_{0}\left(I_{n}-z A_{0}\right)^{-1} \Gamma\right) \Delta^{-1}, \quad \text { where } \Delta=\left(R_{0}-\Gamma^{*} Q \Gamma\right)^{1 / 2} .
$$

The following result is an addition to Lemma 2.2.

Lemma 3.3. Let $G$ be given by (1.5) with $A$ stable, and let $P$ be the unique solution of the Stein equation (3.4). Put $R(z)=G(z) G^{*}(z)$, and assume that $T_{R}$ is invertible, or equivalently, assume that the Riccati equation (3.9) has a stabilizing solution $Q$. Then the $n \times n$ matrix $P Q$ has all its eigenvalues in the closed interval $[0,1]$, and

$$
\operatorname{dim} \operatorname{Ker} T_{G}^{*}=\operatorname{dim} \operatorname{Ker}\left(I_{n}-P Q\right) .
$$

Proof. Recall that $H_{G}=W_{o b s} W_{c o n}$ and $Q=W_{o b s}^{*} T_{R}^{-1} W_{o b s}$; see (3.3) and (3.10). Using these identities we see that

$$
H_{G}^{*} T_{R}^{-1} H_{G}=W_{c o n}^{*} W_{o b s}^{*} T_{R}^{-1} W_{\text {obs }} W_{c o n}=W_{c o n}^{*} Q W_{c o n} .
$$


Next we use $P=W_{\text {con }} W_{\text {con }}^{*}$ and the identity (2.5). It follows that

$$
\begin{aligned}
\operatorname{dim} \operatorname{Ker} T_{G}^{*} & =\operatorname{dim} \operatorname{Ker}\left(I-H_{G}^{*} T_{R}^{-1} H_{G}\right)=\operatorname{dim} \operatorname{Ker}\left(I_{n}-W_{c o n}^{*} Q W_{c o n}\right) \\
& =\operatorname{dim} \operatorname{Ker}\left(I_{n}-W_{c o n} W_{c o n}^{*} Q\right)=\operatorname{dim} \operatorname{Ker}\left(I_{n}-P Q\right) .
\end{aligned}
$$

This proves (3.15). By Lemma 2.2 the spectral radius of $I-H_{G}^{*} T_{R}^{-1} H_{G}$ is at most one. Hence (3.16) yields

$$
1 \geq r_{\text {spec }}\left(I-W_{\text {con }}^{*} Q W_{\text {con }}\right)=r_{\text {spec }}\left(I_{n}-P Q\right) .
$$

Finally, note that the non-zero eigenvalues of $P Q$ are equal to the non-zero eigenvalues of $P^{1 / 2} Q P^{1 / 2}$. But the latter matrix is nonnegative (because $Q$ is nonnegative by (3.10)), and thus all the eigenvalues of $P Q$ belong to [0,1], as desired.

The following lemma will be useful in the next sections.

Lemma 3.4. Let $G$ be given by (1.5) with $A$ stable, and let $P$ be the unique solution of the Stein equation (3.4). Assume that $R(z)=G(z) G^{*}(z)$ is positive definite for each $z$ on $\mathbb{T}$, and let $Q$ be the stabilizing solution of the Riccati equation (3.9). Then the following identities hold:

$$
\begin{aligned}
G^{*}(z) C_{0}\left(I_{n}-z A_{0}\right)^{-1} & =C_{1}\left(I_{n}-z A_{0}\right)^{-1}+B^{*}\left(z I_{n}-A^{*}\right)^{-1} Q, \\
G(z) C_{1}\left(I_{n}-z A_{0}\right)^{-1} & =C\left(I_{n}-z A\right)^{-1}\left(I_{n}-P Q\right), \\
R(z) C_{0}\left(I_{n}-z A_{0}\right)^{-1} & =C\left(I_{n}-z A\right)^{-1}+\Gamma^{*}\left(z I_{n}-A^{*}\right)^{-1} Q .
\end{aligned}
$$

Here $A_{0}$ and $C_{0}$ are given by (3.11), the matrix $\Gamma$ is defined by the second identity in (3.6), and $C_{1}$ is given by

$$
C_{1}=D^{*} C_{0}+B^{*} Q A_{0}
$$

Furthermore, we have

$$
\begin{aligned}
B C_{1} & =A\left(I_{n}-P Q\right)-\left(I_{n}-P Q\right) A_{0}, \\
D C_{1} & =C\left(I_{n}-P Q\right), \\
C_{1}^{*} C_{1} & =(Q-Q P Q)-A_{0}^{*}(Q-Q P Q) A_{0} .
\end{aligned}
$$

Proof. We begin the proof with the last three identities and then we proceed with the first three. Using the definition of $A_{0}$ and $C_{0}$ in (3.11) together with the fact that $Q$ is a hermitian matrix satisfying (3.9) we see that

$$
Q=A^{*} Q A_{0}+C^{*} C_{0}
$$

The latter identity will play an important role in deriving (3.17) and (3.23). 
Proof of (3.21). Using the definition of $C_{1}$ in (3.20) and the Stein equation (3.4), we have

$$
\begin{aligned}
B C_{1} & =B D^{*} C_{0}+B B^{*} Q A_{0}=B D^{*} C_{0}+\left(P-A P A^{*}\right) Q A_{0} \\
& =B D^{*} C_{0}+P Q A_{0}-A P A^{*} Q A_{0} \\
& =B D^{*} C_{0}+P Q A_{0}-A P\left(Q-C^{*} C_{0}\right) \quad[\mathrm{by}(3.24)] \\
& =\left(B D^{*}+A P C^{*}\right) C_{0}+P Q A_{0}-A P Q \\
& =\Gamma C_{0}+P Q A_{0}-A P Q=A-A_{0}+P Q A_{0}-A P Q \\
& =A\left(I_{n}-P Q\right)-\left(I_{n}-P Q\right) A_{0} .
\end{aligned}
$$

Proof of (3.22). Notice that

$$
\begin{aligned}
D C_{1} & =D D^{*} C_{0}+D B^{*} Q A_{0} \\
& =D D^{*} C_{0}+\left(\Gamma^{*}-C P A^{*}\right) Q A_{0} \quad[\text { by the second identity in (3.6)] } \\
& =D D^{*} C_{0}+\Gamma^{*} Q A_{0}-C P A^{*} Q A_{0} \\
& =D D^{*} C_{0}+\Gamma^{*} Q\left(A-\Gamma C_{0}\right)-C P\left(Q-C^{*} C_{0}\right) \\
& =\left(D D^{*}+C P C^{*}\right) C_{0}+\Gamma^{*} Q A-\Gamma^{*} Q \Gamma C_{0}-C P Q \\
& =\left(R_{0}-\Gamma^{*} Q \Gamma\right) C_{0}+\Gamma^{*} Q A-C P Q \quad[\text { by the first identity in (3.6) } \\
& =C-\Gamma^{*} Q A+\Gamma^{*} Q A-C P Q \quad[\text { by the second identity in (3.11)] } \\
& =C\left(I_{n}-P Q\right) .
\end{aligned}
$$

Proof of (3.23). We use $C_{1}^{*}=C_{0}^{*} D+A_{0}^{*} Q B$ and the previous identities for $B C_{1}$ and $D C_{1}$ above. This yields

$$
\begin{aligned}
C_{1}^{*} C_{1} & =C_{0}^{*} D C_{1}+A_{0}^{*} Q B C_{1} \\
& =C_{0}^{*} C\left(I_{n}-P Q\right)+A_{0}^{*} Q\left(A\left(I_{n}-P Q\right)-\left(I_{n}-P Q\right) A_{0}\right) \\
& =\left(C_{0}^{*} C+A_{0}^{*} Q A\right)\left(I_{n}-P Q\right)-A_{0}^{*} Q\left(I_{n}-P Q\right) A_{0} \\
& =Q\left(I_{n}-P Q\right)-A_{0}^{*} Q\left(I_{n}-P Q\right) A_{0} \quad[\text { by }(3.24)] \\
& =Q-Q P Q-A_{0}^{*}(Q-Q P Q) A_{0} .
\end{aligned}
$$

Proof of (3.17). Using the representation of $G(z)$ given by (1.5), we obtain

$$
\begin{aligned}
G^{*}(z) C_{0}\left(I_{n}-z A_{0}\right)^{-1}= & D^{*} C_{0}\left(I_{n}-z A_{0}\right)^{-1} \\
& +B^{*}\left(z I_{n}-A^{*}\right)^{-1} C^{*} C_{0}\left(I_{n}-z A_{0}\right)^{-1}
\end{aligned}
$$

According to (3.24), we have $C^{*} C_{0}=Q-A^{*} Q A_{0}$. It follows that

$$
C^{*} C_{0}=\left(z I_{n}-A^{*}\right) Q A_{0}+Q\left(I_{n}-z A_{0}\right) .
$$

This yields

$$
\left(z I_{n}-A^{*}\right)^{-1} C^{*} C_{0}\left(I_{n}-z A_{0}\right)^{-1}=Q A_{0}\left(I_{n}-z A_{0}\right)^{-1}+\left(z I_{n}-A^{*}\right)^{-1} Q .
$$


By using the latter identity in the formula for $G^{*}(z) C_{0}\left(I_{n}-z A_{0}\right)^{-1}$ above we obtain

$$
\begin{aligned}
G^{*}(z) C_{0}\left(I_{n}-z A_{0}\right)^{-1}= & D^{*} C_{0}\left(I_{n}-z A_{0}\right)^{-1} \\
& +B^{*} Q A_{0}\left(I_{n}-z A_{0}\right)^{-1}+B^{*}\left(z I_{n}-A^{*}\right)^{-1} Q .
\end{aligned}
$$

As $C_{1}=D^{*} C_{0}+B^{*} Q A_{0}$, we have proved (3.17).

Proof of (3.18). Note that

$$
\begin{aligned}
G(z) C_{1}\left(I_{n}-z A_{0}\right)^{-1}= & D C_{1}\left(I_{n}-z A_{0}\right)^{-1} \\
& +z C\left(I_{n}-z A\right)^{-1} B C_{1}\left(I_{n}-z A_{0}\right)^{-1} .
\end{aligned}
$$

Using (3.21) we have

$$
\begin{aligned}
z B C_{1} & =z A\left(I_{n}-P Q\right)-z\left(I_{n}-P Q\right) A_{0} \\
& =\left(I_{n}-P Q\right)\left(I_{n}-z A_{0}\right)-\left(I_{n}-z A\right)\left(I_{n}-P Q\right) .
\end{aligned}
$$

This yields

$$
\begin{aligned}
& z\left(I_{n}-z A\right)^{-1} B C_{1}\left(I_{n}-z A_{0}\right)^{-1} \\
& \quad=\left(I_{n}-z A\right)^{-1}\left(I_{n}-P Q\right)-\left(I_{n}-P Q\right)\left(I_{n}-z A_{0}\right)^{-1} .
\end{aligned}
$$

Thus

$$
\begin{aligned}
G(z) C_{1}\left(I_{n}-z A_{0}\right)^{-1}= & D C_{1}\left(I_{n}-z A_{0}\right)^{-1} \\
& +C\left(I_{n}-z A\right)^{-1}\left(I_{n}-P Q\right) \\
& -C\left(I_{n}-P Q\right)\left(I_{n}-z A_{0}\right)^{-1} .
\end{aligned}
$$

Now (3.22) shows that $D C_{1}\left(I_{n}-z A_{0}\right)^{-1}-C\left(I_{n}-P Q\right)\left(I_{n}-z A_{0}\right)^{-1}=0$. Thus (3.18) holds.

Proof of (3.19). Using (3.17) and (3.18) we have

$$
\begin{aligned}
R(z) C_{0}\left(I_{n}-z A_{0}\right)^{-1} & =G(z) G^{*}(z) C_{0}\left(I_{n}-z A_{0}\right)^{-1} \\
& =G(z) C_{1}\left(I_{n}-z A_{0}\right)^{-1}+G(z) B^{*}\left(z I_{n}-A^{*}\right)^{-1} Q \\
& =C\left(I_{n}-z A\right)^{-1}\left(I_{n}-P Q\right)+G(z) B^{*}\left(z I_{n}-A^{*}\right)^{-1} Q .
\end{aligned}
$$

Inserting the identity for $G(z) B^{*}\left(z I_{n}-A^{*}\right)^{-1}$ given by (3.8) into (3.25) we obtain (3.19).

\section{Proof of Theorem 1.1}

It will be convenient to prove the following result first.

Theorem 4.1. Let $G$ be given by (1.5) with $A$ stable, and let $P$ be the unique solution of the Stein equation (3.4). Then the operator $T_{G}$ is right invertible if and only if

(i) the Riccati equation (3.9) has a stabilizing solution $Q$ and

(ii) the matrix $I_{n}-P Q$ is non-singular. 
In that case the operator $T_{G} T_{G}^{*}$ is invertible and its inverse is given by

$$
\left(T_{G} T_{G}^{*}\right)^{-1}=T_{\Psi} T_{\Psi}^{*}+K\left(I_{n}-P Q\right)^{-1} P K^{*} .
$$

Here $T_{\Psi}$ is the block lower triangular Toeplitz operator on $\ell_{+}^{2}\left(\mathbb{C}^{m}\right)$ defined by the stable rational matrix function (3.14), and $K$ is the observability operator defined by

$$
K=W_{0, o b s}=\left[\begin{array}{c}
C_{0} \\
C_{0} A_{0} \\
C_{0} A_{0}^{2} \\
\vdots
\end{array}\right]: \mathbb{C}^{n} \rightarrow \ell_{+}^{2}\left(\mathbb{C}^{m}\right) .
$$

In that case $\Xi=\mathcal{F}_{\mathbb{C}^{m}}\left(T_{G} T_{G}^{*}\right)^{-1} \tilde{E}$ is a stable rational $m \times m$ matrix function, and $\Xi$ admits the following state space representation:

$$
\Xi(z)=D_{0}+z C_{0}\left(I_{n}-z A_{0}\right)^{-1} B_{0},
$$

where $A_{0}$ and $C_{0}$ are given by (3.11), and

$$
\begin{aligned}
& B_{0}=A_{0}\left(I_{n}-P Q\right)^{-1} P C_{0}^{*}-\Gamma\left(R_{0}-\Gamma^{*} Q \Gamma\right)^{-1}, \\
& D_{0}=C_{0}\left(I_{n}-P Q\right)^{-1} P C_{0}^{*}+\left(R_{0}-\Gamma^{*} Q \Gamma\right)^{-1} .
\end{aligned}
$$

Finally, it is noted that $D_{0}$ is strictly positive.

Proof. By Proposition 2.1 and Lemma 2.2 the operator $T_{G}$ is right invertible if and only if $T_{R}$ is invertible and $\operatorname{dim} \operatorname{Ker} T_{G}^{*}=0$. But $T_{R}$ being invertible is equivalent to the requirement that the Riccati equation (3.9) has a stabilizing solution $Q$, and in that case, Lemma 2.2 tells us that $\operatorname{dim} \operatorname{Ker} T_{G}^{*}=0$ if and only if $I_{n}-P Q$ is non-singular. This proves the necessity and sufficiency of the conditions (i) and (ii).

Now, assume that these two conditions are fulfilled. Then we know that $T_{G} T_{G}^{*}$ is invertible and its inverse is given by (2.4). We have to transform (2.4) into (4.1). Note that (3.19) tells us that $T_{R} W_{0, o b s}=W_{o b s}$. It follows that

$$
T_{R}^{-1} H_{G}=T_{R}^{-1} W_{\text {obs }} W_{c o n}=W_{0, o b s} W_{c o n} .
$$

We already know that $H_{G}^{*} T_{R}^{-1} H_{G}=W_{c o n}^{*} Q W_{\text {con }}$; see (3.16). Since $P=$ $W_{\text {con }} W_{\text {con }}^{*}$, we obtain

$$
\begin{aligned}
& T_{R}^{-1} H_{G}\left(I-H_{G}^{*} T_{R}^{-1} H_{G}\right)^{-1} H_{G}^{*} T_{R}^{-1} \\
& \quad=W_{0, o b s} W_{c o n}\left(I-W_{c o n}^{*} Q W_{c o n}\right)^{-1} W_{c o n}^{*} W_{0, o b s}^{*} \\
& \quad=W_{0, o b s}\left(I_{n}-W_{c o n} W_{c o n}^{*} Q\right)^{-1} W_{c o n} W_{c o n}^{*} W_{0, o b s}^{*} \\
& \quad=W_{0, o b s}\left(I_{n}-P Q\right)^{-1} P W_{0, o b s}^{*} \\
& \quad=K\left(I_{n}-P Q\right)^{-1} P K^{*} .
\end{aligned}
$$

This takes care of the second term in the right hand side of (4.1). The first term in the right hand side of (4.1) follows by applying Theorem 3.2 to the first term in the right hand side of (2.4). 
It remains to derive the formula for $\Xi=\mathcal{F}_{\mathbb{C}^{m}}\left(T_{G} T_{G}^{*}\right)^{-1} \tilde{E}$. To do this we use (4.1). From (4.2) is clear that $K^{*} \tilde{E}=C_{0}^{*}$. We conclude that

$$
\begin{aligned}
\left(\mathcal{F}_{\mathbb{C}^{m}} K\left(I_{n}-P Q\right)^{-1} P K^{*} \tilde{E}\right)(z)= & \left(\mathcal{F}_{\mathbb{C}^{m}} K\left(I_{n}-P Q\right)^{-1} P C_{0}^{*}\right)(z) \\
= & C_{0}\left(I_{n}-z A_{0}\right)^{-1}\left(I_{n}-P Q\right)^{-1} P C_{0}^{*} \\
= & C_{0}\left(I_{n}-P Q\right)^{-1} P C_{0}^{*}+ \\
& +z C_{0}\left(I_{n}-z A_{0}\right)^{-1} A_{0}\left(I_{n}-P Q\right)^{-1} P C_{0}^{*} .
\end{aligned}
$$

Now consider $\mathcal{F}_{\mathbb{C}^{m}} T_{\Psi} T_{\Psi}^{*} \tilde{E}$. Since $T_{\Psi}^{*}$ is block upper triangular with the matrix $\left(R_{0}-\Gamma^{*} Q \Gamma\right)^{-1 / 2}$ on the main diagonal, $T_{\Psi}^{*} \tilde{E}=\tilde{E}\left(R_{0}-\Gamma^{*} Q \Gamma\right)^{-1 / 2}$. Finally, because $T_{\Psi}$ is the block Toeplitz operator defined by $\Psi$, we obtain

$$
\begin{aligned}
\left(\mathcal{F}_{\mathbb{C}^{m}} T_{\Psi} T_{\Psi}^{*} \tilde{E}\right)(z) & =\Psi(z)\left(R_{0}-\Gamma^{*} Q \Gamma\right)^{-1 / 2} \\
& =\left(R_{0}-\Gamma^{*} Q \Gamma\right)^{-1}-z C_{0}\left(I_{n}-z A_{0}\right)^{-1} \Gamma\left(R_{0}-\Gamma^{*} Q \Gamma\right)^{-1} .
\end{aligned}
$$

By adding (4.6) and (4.7) we see that $\Xi=\mathcal{F}\left(T_{G} T_{G}^{*}\right)^{-1} \tilde{E}$ has the desired state space representation.

To complete the proof, it is noted that

$$
C_{0}\left(I_{n}-P Q\right)^{-1} P C_{0}^{*}=C_{0} P^{1 / 2}\left(I_{n}-P^{1 / 2} Q P^{1 / 2}\right)^{-1} P^{1 / 2} C_{0}^{*}
$$

is positive. Since $\left(R_{0}-\Gamma^{*} Q \Gamma\right)^{-1}$ is strictly positive, it follows that $D_{0}$ is strictly positive.

Corollary 4.2. Let $G$ be given by (1.5) with A stable. Then $M_{G}$ is right invertible if and only if $G$ can be written as $G(z)=D V(z)$, where $D=G(0)$ has full row rank and $V$ is an invertible outer stable rational matrix function. Moreover, in that case one can take for $V$ the function given by

$$
V(z)=I_{p}+z C_{1}\left(I_{n}-P Q\right)^{-1}\left(I_{n}-z A\right)^{-1} B .
$$

Here $P$ and $Q$ are as in Theorem 4.1 and $C_{1}$ is defined by (3.20).

Proof. Assume $G(z)=D V(z)$ for some invertible outer stable rational matrix function $V$, and let $D^{+}$be any right inverse of $D$. Put $U(z)=V(z)^{-1} D^{+}$. Then $G(z) U(z)=D V(z) V(z)^{-1} D^{+}=I_{m}$ for each $|z| \leq 1$. Thus $M_{G} M_{U}=I$, and $M_{G}$ is right invertible.

Conversely, assume $M_{G}$ is right invertible. Let $P$ and $Q$ be as in Theorem 4.1. Then $I_{n}-P Q$ is invertible. Let $V$ be defined by (4.8). By consulting (3.22), we obtain $C=D C_{1}\left(I_{n}-P Q\right)^{-1}$. Thus

$$
\begin{aligned}
G(z) & =D+z C\left(I_{n}-z A\right)^{-1} B \\
& =D+z D C_{1}\left(I_{n}-P Q\right)^{-1}\left(I_{n}-z A\right)^{-1} B \\
& =D V(z) .
\end{aligned}
$$

It remains to show that $V$ is invertible outer. We have

$$
V(z)^{-1}=I_{p}-z C_{1}\left(I_{n}-P Q\right)^{-1}\left(I_{n}-z A^{\times}\right)^{-1} B,
$$


where

$$
\begin{aligned}
A^{\times} & =A-B C_{1}\left(I_{n}-P Q\right)^{-1} \\
& =A-\left(A\left(I_{n}-P Q\right)-\left(I_{n}-P Q\right) A_{0}\right)\left(I_{n}-P Q\right)^{-1} \quad[\text { by }(3.21)] \\
& =\left(I_{n}-P Q\right) A_{0}\left(I_{n}-P Q\right)^{-1} .
\end{aligned}
$$

Therefore $A^{\times}$is similar to the stable matrix $A_{0}$, and hence $A^{\times}$is stable. It follows that both $V(z)$ and $V(z)^{-1}$ are stable rational matrix functions. Thus $V$ is invertible outer.

Proof of Theorem 1.1. In view of Theorem 4.1 we only have to derive the formula for $X=M_{G}^{*}\left(M_{G} M_{G}^{*}\right)^{-1} E$ and to prove the statements in the final paragraph of the theorem.

From (2.3) we see that $M_{G}^{*}\left(M_{G} M_{G}^{*}\right)^{-1} E=\mathcal{F}_{\mathbb{C}^{p}} T_{G}^{*}\left(T_{G} T_{G}^{*}\right)^{-1} \tilde{E}$. It follows that $X=\mathcal{F}_{\mathbb{C}^{p}} T_{G}^{*} \tilde{\Xi}$, where $\tilde{\Xi}=\left(T_{G} T_{G}^{*}\right)^{-1} \tilde{E}$. Put $\Xi=\mathcal{F}_{\mathbb{C}^{m}} \tilde{\Xi}$. According to Theorem 4.1, the function $\Xi$ is given by (4.3). Note that

$$
X=\mathcal{F}_{\mathbb{C}^{p}} T_{G^{*}} \tilde{\Xi}=\mathcal{F}_{\mathbb{C}^{p}} T_{G^{*} \Xi} \tilde{E} .
$$

Lets us compute $G^{*}(z) \Xi(z)$. Using the state space representation (1.5) for $G$ and the identity (3.17) we have

$$
\begin{aligned}
G^{*}(z) \Xi(z)= & G^{*}(z) D_{0}+z G^{*}(z) C_{0}\left(I_{n}-z A_{0}\right)^{-1} B_{0} \\
= & D^{*} D_{0}+B^{*}\left(z I_{n}-A_{0}^{*}\right)^{-1} C^{*} D_{0} \\
& +z C_{1}\left(I_{n}-z A_{0}\right)^{-1} B_{0}+z B^{*}\left(z I_{n}-A^{*}\right)^{-1} Q B_{0} \\
= & D^{*} D_{0}+B^{*} Q B_{0}+z C_{1}\left(I_{n}-z A_{0}\right)^{-1} B_{0} \\
& +B^{*}\left(z I_{n}-A_{0}^{*}\right)^{-1} C^{*} D_{0}+B^{*}\left(z I_{n}-A^{*}\right)^{-1} A^{*} Q B_{0} .
\end{aligned}
$$

It follows that

$$
X(z)=\left(\mathcal{F}_{\mathbb{C}^{p}} T_{G^{*} \Xi} \tilde{E}\right)(z)=D^{*} D_{0}+B^{*} Q B_{0}+z C_{1}\left(I_{n}-z A_{0}\right)^{-1} B_{0} .
$$

Recall that the operators $D_{0}$ and $B_{0}$ are given by (4.5) and (4.4), respectively. Since $C_{1}=D^{*} C_{0}+B^{*} Q A_{0}$, it is clear that $D^{*} D_{0}+B^{*} Q B_{0}=D_{1}$, where $D_{1}$ is defined in Theorem 1.1.

The next step is to show that $B_{0}=-\left(I_{n}-P Q\right)^{-1} B D_{1}$. To accomplish this we compute $B D_{1}$. Let us set $\Lambda=\left(R_{0}-\Gamma^{*} Q \Gamma\right)^{-1}$. Then

$$
\begin{aligned}
B D_{1} & =B\left(D^{*}-B^{*} Q \Gamma\right) \Lambda+B C_{1}\left(I_{n}-P Q\right)^{-1} P C_{0}^{*} \\
& =B D^{*} \Lambda-B B^{*} Q \Gamma \Lambda+B C_{1}\left(I_{n}-P Q\right)^{-1} P C_{0}^{*} \\
& =B D^{*} \Lambda-P Q \Gamma \Lambda+A P A^{*} Q \Gamma \Lambda+B C_{1}\left(I_{n}-P Q\right)^{-1} P C_{0}^{*} \\
& =\left(I_{n}-P Q\right) \Gamma \Lambda+\left(B D^{*}-\Gamma\right) \Lambda+A P A^{*} Q \Gamma \Lambda+B C_{1}\left(I_{n}-P Q\right)^{-1} P C_{0}^{*} .
\end{aligned}
$$


We proceed with

$$
\begin{aligned}
& \left(B D^{*}-\Gamma\right) \Lambda+A P A^{*} Q \Gamma \Lambda+B C_{1}\left(I_{n}-P Q\right)^{-1} P C_{0}^{*} \\
& \quad=-A P C^{*} \Lambda+A P A^{*} Q \Gamma \Lambda+B C_{1}\left(I_{n}-P Q\right)^{-1} P C_{0}^{*} \\
& \quad=-A P C_{0}^{*}+B C_{1}\left(I_{n}-P Q\right)^{-1} P C_{0}^{*} \\
& \quad=\left(B C_{1}\left(I_{n}-P Q\right)^{-1}-A\right) P C_{0}^{*} \\
& \quad=\left(B C_{1}-A\left(I_{n}-P Q\right)\right)\left(I_{n}-P Q\right)^{-1} P C_{0}^{*} \quad[\text { by }(3.21)] \\
& \quad=\left(A\left(I_{n}-P Q\right)-A\left(I_{n}-P Q\right)-\left(I_{n}-P Q\right) A_{0}\right)\left(I_{n}-P Q\right)^{-1} P C_{0}^{*} \\
& \quad=-\left(I_{n}-P Q\right) A_{0}\left(I_{n}-P Q\right)^{-1} P C_{0}^{*} .
\end{aligned}
$$

Thus

$$
\begin{aligned}
B D_{1} & =\left(I_{n}-P Q\right) \Gamma \Lambda-\left(I_{n}-P Q\right) A_{0}\left(I_{n}-P Q\right)^{-1} P C_{0}^{*} \\
& =-\left(I_{n}-P Q\right)\left(-\Gamma \Lambda+A_{0}\left(I_{n}-P Q\right)^{-1} P C_{0}^{*}\right) \\
& =-\left(I_{n}-P Q\right) B_{0} .
\end{aligned}
$$

We conclude with the statements in the final paragraph of the theorem. First we prove the result about McMillan degrees. To do this assume that the number $n$ in the state space representation (1.5) is chosen as small as possible. In that case, $\delta(G)=n$. Since the matrix $A_{0}$ in the state space representation of $X$ has the same size as $A$, we conclude that $\delta(X) \leq n$. Thus $\delta(X) \leq \delta(G)$, as desired.

Finally, we prove (1.10). The left hand side of (1.10) can be written as $D_{1}^{*} N D_{1}$, where

$$
N=I_{p}+B^{*}\left(I_{n}-Q P\right)^{-1}\left(\sum_{\nu=0}^{\infty}\left(A_{0}^{*}\right)^{\nu} C_{1}^{*} C_{1} A_{0}^{\nu}\right)\left(I_{n}-P Q\right)^{-1} B
$$

From (3.23) we know that $\sum_{\nu=0}^{\infty}\left(A_{0}^{*}\right)^{\nu} C_{1}^{*} C_{1} A_{0}^{\nu}=Q-Q P Q$. It follows that

$$
\begin{aligned}
N & =I_{p}+B^{*}\left(I_{n}-Q P\right)^{-1}(Q-Q P Q)\left(I_{n}-P Q\right)^{-1} B \\
& =I_{p}+B^{*} Q\left(I_{n}-P Q\right)^{-1} B .
\end{aligned}
$$

Thus $D_{1}^{*} N D_{1}$ is equal to the right side of (1.10).

A direct proof that $X$ is a solution of (1.1). Let $X$ be as in Theorem 1.1. From our construction of $X$ we know that $X$ is a solution of (1.1). This fact can also be checked directly by using (3.18) and (3.22). To see this, recall that $X$ is given by (1.9). By using (3.18) we compute that

$$
\begin{aligned}
G(z) X(z) & =G(z) D_{1}-z G(z) C_{1}\left(I_{n}-z A_{0}\right)^{-1}\left(I_{n}-P Q\right)^{-1} B D_{1} \\
& =D D_{1}+z C\left(z I_{n}-A\right)^{-1} B D_{1}-z C\left(z I_{n}-A\right)^{-1} B D_{1} \\
& =D D_{1} .
\end{aligned}
$$


It remains to show $D D_{1}=I_{m}$. For this purpose we use (3.22). As before put $\Lambda=\left(R_{0}-\Gamma^{*} Q \Gamma\right)^{-1}$. We compute

$$
\begin{aligned}
D D_{1} & =\left(D D^{*}-D B^{*} Q \Gamma\right) \Lambda+D C_{1}(I-P Q)^{-1} P C_{0}^{*} \\
& =\left(D D^{*}-D B^{*} Q \Gamma\right) \Lambda+C P C_{0}^{*} \\
& =\left(D D^{*}-\Gamma^{*} Q \Gamma+C P A^{*} Q \Gamma\right) \Lambda+C P\left(C^{*}-A^{*} Q \Gamma\right) \Lambda \\
& =\left(D D^{*}+C P C^{*}-\Gamma^{*} Q \Gamma\right) \Lambda \\
& =\left(R_{0}-\Gamma^{*} Q \Gamma\right) \Lambda=I_{m} .
\end{aligned}
$$

Hence $D D_{1}=I_{m}$, and $G(z) X(z)=I_{m}$.

\section{Two Examples}

In this section we present two examples. The first is a simple example for which all computations can be carried out by hand. For the second example we use MatLab procedures to obtain the desired formulas.

Example 1. Consider the $1 \times 2$ matrix function $G(z)=\left[\begin{array}{ll}1+z & -z\end{array}\right]$. Obviously,

$$
\left[\begin{array}{ll}
1+z & -z
\end{array}\right]\left[\begin{array}{l}
1 \\
1
\end{array}\right]=1
$$

Hence the equation $G(z) X(z)=1$ has a stable rational matrix solution. The solution $\left[\begin{array}{ll}1 & 1\end{array}\right]^{\mathrm{T}}$ in the above equation is not the least squares solution but it is the optimal corona solution (that is, the solution of minimal $H^{\infty}$ norm); see [29]. We shall use Theorem 1.1 to compute the least squares solution.

A minimal realization of $G$ is given by

$$
A=0, \quad B=\left[\begin{array}{ll}
1 & -1
\end{array}\right], \quad C=1, \quad D=\left[\begin{array}{ll}
1 & 0
\end{array}\right] .
$$

Solving the symmetric Stein equation (3.4) for this case, we see that $P=2$. Since $G(z) G^{*}(z)=3+z+z^{-1}$, we have $R_{0}=3$ and $\Gamma=1$. The Riccati equation (3.9) now becomes

$$
Q=\frac{1}{3-Q},
$$

and the stabilizing solution is given by $q=\frac{1}{2}(3-\sqrt{5})$. We see that $q P=3-\sqrt{5}$ is in the open unit disc, as expected.

Inserting this data into the formulas for $C_{0}$ and $A_{0}$ in (3.11), we obtain $C_{0}=q$ and $A_{0}=-q$. Computing $C_{1}$ and $D_{1}$ from Theorem 1.1, and using the fact that $q=1 /(3-q)$, we arrive at

$$
\begin{aligned}
C_{1} & =\left[\begin{array}{l}
q \\
0
\end{array}\right]-\left[\begin{array}{c}
1 \\
-1
\end{array}\right] q^{2}=q\left[\begin{array}{c}
1-q \\
q
\end{array}\right] \\
D_{1} & =\left(\left[\begin{array}{l}
1 \\
0
\end{array}\right]-\left[\begin{array}{c}
1 \\
-1
\end{array}\right] q\right) q+q\left[\begin{array}{c}
1-q \\
q
\end{array}\right] \frac{2}{1-2 q} q=\frac{q}{1-2 q}\left[\begin{array}{c}
1-q \\
q
\end{array}\right]
\end{aligned}
$$

It follows that $-(1-P q)^{-1} B D_{1}=-(1-P q)^{-1} q=-q(1-2 q)^{-1}$. Using Theorem 1.1, we see that for this case the least squares solution $X$ of (1.1) 
is given by

$$
\begin{aligned}
X(z) & =D_{1}-\frac{z}{1+z q} C_{1}(1-P q)^{-1} B D_{1} \\
& =\frac{q}{1-2 q}\left[\begin{array}{c}
1-q \\
q
\end{array}\right]-\frac{z}{1+z q}\left(\frac{q^{2}}{1-2 q}\right)\left[\begin{array}{c}
1-q \\
q
\end{array}\right] \\
& =\frac{1}{1-2 q}\left[\begin{array}{c}
1-q \\
q
\end{array}\right]\left(q-\frac{q^{2} z}{1+z q}\right) .
\end{aligned}
$$

In other words,

$$
X(z)=\frac{q}{1-2 q}\left[\begin{array}{c}
1-q \\
q
\end{array}\right](1+z q)^{-1}, \quad \text { where } q=\frac{1}{2}(3-\sqrt{5}) .
$$

Let us check directly that $X$ is indeed a solution of (1.1):

$$
\begin{aligned}
{[1+z-z] X(z) } & =\frac{q}{1-2 q}((1+z)(1-q)-z q)(1+z q)^{-1} \\
& =\frac{q}{1-2 q}(1+z-q-2 q z)(1+z q)^{-1} \\
& =\frac{q}{1-2 q}((1-2 q) z+(1-q))(1+z q)^{-1} \\
& =q z(1+z q)^{-1}+\frac{q-q^{2}}{1-2 q}(1+z q)^{-1}=1 .
\end{aligned}
$$

The last equality holds because $\left(q-q^{2}\right) /(1-2 q)=1$. To obtain this identity recall that $q$ satisfies $q=1 /(3-q)$ or $q^{2}-3 q+1=0$.

Example 2. Consider the $2 \times 3$ matrix function $G(z)$ given by

$$
G(z)=\left[\begin{array}{ccc}
1 & z+z^{2} & z^{2} \\
0 & 1+z & z
\end{array}\right]
$$

We have

$$
\left[\begin{array}{lll}
1 & z+z^{2} & z^{2} \\
0 & 1+z & z
\end{array}\right]\left[\begin{array}{ll}
1 & -z \\
0 & 1 \\
0 & -1
\end{array}\right]=I_{2} .
$$

Hence the equation $G(z) X(z)=I_{2}$ has a stable rational matrix solution. Our aim is to compute the least squares solution. To do this we apply the method provided by Theorem 1.1.

A minimal realization for $G$ is given by

$$
A=\left[\begin{array}{ll}
0 & 1 \\
0 & 0
\end{array}\right], \quad B=\left[\begin{array}{lll}
0 & 1 & 0 \\
0 & 1 & 1
\end{array}\right], \quad C=I_{2}, \quad D=\left[\begin{array}{lll}
1 & 0 & 0 \\
0 & 1 & 0
\end{array}\right] .
$$

For this case the solution of the symmetric Stein equation (3.4) is given by

$$
P=\left[\begin{array}{ll}
3 & 1 \\
1 & 2
\end{array}\right] .
$$


Furthermore, one computes that

$$
\begin{gathered}
R_{0}=D D^{*}+C P C^{*}=\left[\begin{array}{ll}
1 & 0 \\
0 & 1
\end{array}\right]+\left[\begin{array}{ll}
3 & 1 \\
1 & 2
\end{array}\right]=\left[\begin{array}{ll}
4 & 1 \\
1 & 3
\end{array}\right], \\
\Gamma=B D^{*}+A P C^{*}=\left[\begin{array}{ll}
0 & 1 \\
0 & 1
\end{array}\right]+\left[\begin{array}{ll}
1 & 2 \\
0 & 0
\end{array}\right]=\left[\begin{array}{ll}
1 & 3 \\
0 & 1
\end{array}\right] .
\end{gathered}
$$

Since in this case all matrices are real, the unique stabilizing solution $Q$ of the corresponding Riccati equation is real symmetric. Hence (cf., Section 12.7 in [18]) we can assume that $Q$ is of the form

$$
Q=\left[\begin{array}{ll}
q_{1} & q_{2} \\
q_{2} & q_{3}
\end{array}\right]
$$

and one computes that the Riccati equation (3.9) takes the form

$$
\begin{aligned}
{\left[\begin{array}{ll}
q_{1} & q_{2} \\
q_{2} & q_{3}
\end{array}\right]=} & {\left[\begin{array}{ll}
0 & 0 \\
0 & q_{1}
\end{array}\right]+\left[\begin{array}{cc}
1 & 0 \\
-q_{1} & 1-3 q_{1}-q_{2}
\end{array}\right] } \\
& \times\left[\begin{array}{cc}
4-q_{1} & 1-3 q_{1}-q_{2} \\
1-3 q_{1}-q_{2} & 3-9 q_{1}-6 q_{2}-q_{3}
\end{array}\right]^{-1}\left[\begin{array}{cc}
1 & -q_{1} \\
0 & 1-3 q_{1}-q_{2}
\end{array}\right]
\end{aligned}
$$

To find the stabilizing solution by hand is a problem. However we can use the standard MatLab command 'dare' from the MatLab control toolbox to compute the stabilizing solution $Q$ for the case considered here. This yields:

$$
Q=\left[\begin{array}{cc}
0.2764 & -0.1056 \\
-0.1056 & 0.4223
\end{array}\right]
$$

By using this $Q$ in (3.11) we obtain

$$
A_{0}=\left[\begin{array}{ll}
0.0403 & -0.1613 \\
0.1056 & -0.4223
\end{array}\right], \quad C_{0}=\left[\begin{array}{cc}
0.2764 & -0.1056 \\
-0.1056 & 0.4223
\end{array}\right] .
$$

Inserting this data in the formulas of Theorem 1.1 and using MatLab to make the computations we arrive at

$$
\begin{aligned}
C_{1}= & {\left[\begin{array}{cc}
0.2764 & -0.1056 \\
-0.0652 & 0.2610 \\
0.0403 & -0.1613
\end{array}\right], \quad D_{1}=\left[\begin{array}{cc}
1 & 0 \\
0 & 1 \\
0 & -0.6180
\end{array}\right], } \\
& -\left(I_{2}-P Q\right)^{-1} B D_{1}=\left[\begin{array}{cc}
0 & -4.6180 \\
0 & -2.6180
\end{array}\right] .
\end{aligned}
$$

This then shows that the least squares solution $X(z)$ is given by

$$
X(z)=\left[\begin{array}{ll}
1 & -z \\
0 & \frac{1}{1+0.3820 z} \\
0 & \frac{-0.618}{1+0.3820 z}
\end{array}\right] .
$$

Remark on coprime factorization. In this final remark we use Example 2 above to show that the least squares solution (5.6) cannot be derived via 
the double coprime factorization approach in Chapter 4 of [30]. To see this, put

$$
G_{1}(z)=\left[\begin{array}{ll}
1 & z+z^{2} \\
0 & 1+z
\end{array}\right], G_{2}(z)=\left[\begin{array}{l}
z^{2} \\
z
\end{array}\right], P(z)=G_{1}(z)^{-1} G_{2}(z)=\left[\begin{array}{c}
0 \\
\frac{z}{1+z}
\end{array}\right] .
$$

Note that $P(z)=G_{1}(z)^{-1} G_{2}(z)$ is a left coprime factorization. Using the matrices in (5.5), we see that

$$
\begin{gathered}
G_{1}(z)=I_{2}+z C\left(I_{2}-z A\right)^{-1} B_{1}, \quad G_{2}(z)=z C\left(I_{2}-z A\right)^{-1} B_{2}, \\
\text { where } B_{1}=\left[\begin{array}{ll}
0 & 1 \\
0 & 1
\end{array}\right] \text { and } B_{2}=\left[\begin{array}{l}
0 \\
1
\end{array}\right] .
\end{gathered}
$$

Furthermore,

$$
P(z)=z C\left(I_{2}-z A_{1}\right)^{-1} B_{2}, \quad \text { with } A_{1}=A-B_{1} C=\left[\begin{array}{ll}
0 & 0 \\
0 & -1
\end{array}\right] .
$$

Now let us apply the discrete time analogue of the results of Section 4.2 in [30] to this realization of $P(z)$. Choose $K=\left[\begin{array}{ll}k_{1} & k_{2}\end{array}\right]$ such that

$$
A_{2}:=A_{1}+B_{2} K=\left[\begin{array}{cc}
0 & 0 \\
k_{1} & -1+k_{2}
\end{array}\right] \text { is stable. }
$$

Put

$$
H_{1}(z)=I_{2}-z C\left(I_{2}-z A_{2}\right)^{-1} B_{1}, \quad H_{2}(z)=z K\left(I_{2}-z A_{2}\right)^{-1} B_{1} .
$$

Then, according to the discrete time analogue of Theorem 4 in Section 4.2 of [30] (see also Section 21.5.2 in [31]), we have $G_{1}(z) H_{1}(z)+G_{2}(z) H_{2}(z)=I_{2}$. Hence for any choice of $k_{1}$ and $k_{2}$ in (5.7),

$$
H(z):=\left[\begin{array}{l}
H_{1}(z) \\
H_{2}(z)
\end{array}\right]=\left[\begin{array}{ll}
1 & 0 \\
0 & 1 \\
0 & 0
\end{array}\right]+z\left[\begin{array}{cc}
-1 & 0 \\
0 & -1 \\
k_{1} & k_{2}
\end{array}\right]\left[\begin{array}{cc}
1 & 0 \\
z k_{1} & 1+z-z k_{2}
\end{array}\right]^{-1}\left[\begin{array}{ll}
0 & 1 \\
0 & 1
\end{array}\right]
$$

is a stable rational matrix function satisfying $G(z) H(z)=I_{2}$. Moreover, $\delta(H) \leq \delta(G)$. However, for any choice of $k_{1}$ and $k_{2}$ the value of $H$ at zero is different for the value at zero of $X$ given by (5.6). Thus there is no choice of $k_{1}, k_{2}$ such that $H=X$, and hence we cannot obtain the least-squares solution via the above coprime factorization method.

Open Access. This article is distributed under the terms of the Creative Commons Attribution Noncommercial License which permits any noncommercial use, distribution, and reproduction in any medium, provided the original author(s) and source are credited.

\section{References}

[1] Arveson, W.: Interpolation problems in nest algebras. J. Funct. Anal. 20, 208233 (1975) 
[2] Bart, H., Gohberg, I., Kaashoek, M.A., Ran, A.C.M.: Factorization of Matrix and Operator Functions: The State Space Method, OT 178. Birkhäuser Verlag, Basel (2008)

[3] Bart, H., Gohberg, I., Kaashoek, M.A., Ran, A.C.M.: A State Space Approach to Canonical Factorization: Convolution Equations and Mathematical Systems, OT 200. Birkhäuser Verlag, Basel (2010)

[4] Carleson, L.: Interpolation by bounded analytic functions and the corona problem. Ann. Math. 76, 547-559 (1962)

[5] Corless, M.J., Frazho, A.E.: Linear Sytems and Control. Marcel Dekker, New York (2003)

[6] Doyle, J.C., Glover, K., Khargonekar, P.P., Francis, B.A.: State-space solutions to standard $H^{2}$ and $H^{\infty}$ control problems. IEEE Trans. Autom. Control 34, 831-847 (1989)

[7] Frazho, A.E., Bosri, W.: An Operator Perspective on Signals and Systems, OT 204. Birkhäuser Verlag, Basel (2010)

[8] Frazho, A.E., Kaashoek, M.A., Ran, A.C.M.: The non-symmetric discrete algebraic Riccati equation and canonical factorization of rational matrix functions on the unit circle. Integral Equ. Oper. Theory 66, 215-229 (2010)

[9] Frazho, A.E., Kaashoek, M.A., Ran, A.C.M.: Right invertible multiplication operators and stable rational matrix solutions to an associate Bezout equation, II: description of all solutions (in preparation, 2011)

[10] Fuhrmann, P.: On the corona theorem and its applications to spectral problems in Hilbert space. Trans. Am. Math. Soc. 132, 55-66 (1968)

[11] Gohberg, I., Goldberg, S., Kaashoek, M.A.: Classes of Linear Operators, vol. II, OT 63. Birkhäuser Verlag, Basel (1993)

[12] Gohberg, I., Kaashoek, M.A., Lerer, L.: The resultant for regular matrix polynomials and quasi commutativity. Indiana Univ. Math. J. 57, 2783-2813 (2008)

[13] Green, M., Limebeer, D.J.N.: Linear Robust Control. Prentice Hall, Englewood Cliffs (1995)

[14] Heij, Chr., Ran, A.C.M., Schagen, F.van : Introduction to Mathematical Systems Theory. Linear Systems, Identification and Control. Birkhäuser Verlag, Basel (2007)

[15] Helton, J.W.: Operator Theory, Analytic Functions, Matrices and Electrical Engineering, Regional Conference Series in Mathematics, vol. 68. American Mathematical Society, Providence (1987)

[16] Iglesias, P.A., Glover, K.: State-space approach to discrete-time $H^{\infty}$ control. Int. J. Control 54, 1031-1073 (1991)

[17] Iglesias, P.A., Mustafa, D.: State-space solution of the discrete-time minimum entropy control problem via separation. IEEE Trans. Autom. Control 38, 15251530 (1993)

[18] Lancaster, P., Rodman, L.: Algebraic Riccati Equations. Clarendon Press, Oxford (1995)

[19] Nikol'skii, N.K.: Treatise on the Shift Operator, Grundlehren, vol. 273. Springer, Berlin (1986)

[20] Nikol'skii, N.K.: Operators, functions and systems. Math. Surveys Monographs, vol. 92. American Mathematical Society, Providence (2002) 
[21] Peller, V.V.: Hankel Operators and their Applications, Springer Monographs in Mathematics. Springer, Berlin (2003)

[22] Schubert, C.F.: The corona theorem as an operator problem. Proc. Am. Math. Soc. 69, 73-76 (1978)

[23] Stoorvogel, A.A.: The $H_{\infty}$ control problem: a state space approach. Dissertation, Eindhoven University of Technology, Eindhoven (1990)

[24] Sz-Nagy, B., Foias, C.: On contractions similar to isometries and Toeplitz operators. Ann. Acad. Sci. Fenn. Ser. A I Math. 2, 553-564 (1976)

[25] Tolokonnikov, V.A.: Estimates in Carleson's corona theorem. Ideals of the algebra $H^{\infty}$, the problem of Szőkefalvi-Nagy. Zap. Naučn. Sem. Leningrad. Otdel. Mat. Inst. Steklov. (LOMI) 113, 178-198 (Russian) (1981)

[26] Treil, S.: Lower bounds in the matrix corona theorem and the codimension one conjecture. GAFA 14, 1118-1133 (2004)

[27] Treil, S., Wick, B.D.: The matrix-valued $H^{p}$ corona problem in the disk and polydisk. J. Funct. Anal. 226, 138-172 (2005)

[28] Trent, T.T., Zhang, X.: A matricial corona theorem. Proc. Am. Math. Soc. 134, 2549-2558 (2006)

[29] Trent, T.T.: An algorithm for the corona solutions on $H^{\infty}(D)$. Integral Equ. Oper. Theory 59, 421-435 (2007)

[30] Vidyasagar, M.: Control System Synthesis: A Factorization Approach. The MIT Press, Cambridge (1985)

[31] Zhou, K., Doyle, J.C., Glover, K.: Robust and Optimal Control. Prentice Hall, Englewood Cliffs (1996)

\author{
A.E. Frazho \\ Department of Aeronautics and Astronautics \\ Purdue University \\ West Lafayette, IN 47907, USA \\ e-mail: frazho@ecn.purdue.edu \\ M.A. Kaashoek $(\bowtie)$ and A.C.M. Ran \\ Afdeling Wiskunde \\ Faculteit der Exacte Wetenschappen \\ Vrije Universiteit \\ De Boelelaan 1081a \\ 1081 HV Amsterdam \\ The Netherlands \\ e-mail: ma.kaashoek@few.vu.nl; \\ acm.ran@few.vu.nl
}

Received: September 7, 2010.

Revised: April 6, 2011. 